\title{
Clinical and biochemical characteristics of Sjögren's syndrome and its differences from keratoconjunctivitis sicca
}

\author{
Ismail TASKIRAN $^{1}$ (D), Umut KALYONCU ${ }^{2}$ (D), Sedat KIRAZ ${ }^{2}$ (iD \\ ${ }^{1}$ Division of Gastroenterology, Department of Internal Medicine, School of Medicine, Aydın Menderes University, Aydın, Turkey. \\ ${ }^{2}$ Department of Rheumatology, School of Medicine, Hacettepe University, Ankara, Turkey.
}

Corresponding Author: Ismail TASKIRAN

E-mail: dr_istaskiran@hotmail.com

Submitted: 12.01.2021 Accepted: 29.03.2021

\begin{abstract}
Objective: In this study we aimed to compare the clinical and biochemical characteristics of primary Sjögren's syndrome patients with that of keratoconjunctivitis sicca, secondary Sjögren syndrome and undifferentiated disease.

Patients and Methods: Patients with Sjögren's syndrome and keratoconjunctivitis sicca who applied between August 2009 and January 2010 were included in this study. Demographic data, laboratory characteristics, Schirmer's test and salivary gland biopsies of the patients were recorded.

Results: Average diagnosis age for 87 primary Sjögren's syndrome, 25 secondary Sjögren’s syndrome, 27 keratoconjunctivitis sicca and 44 undifferentiated group patients were $45 \pm 13,43 \pm 14,44 \pm 11$ ve $45 \pm 11$ years ( $p>0.05$ ), accordingly. Critical and severe mouth dryness rate was $45 \%$ in primary Sjögren's syndrome group and 7\% in keratoconjunctivitis sicca group, critical and severe eye dryness was $43 \%$ in primary Sjögren's syndrome group and $78 \%$ in keratoconjunctivitis sicca group $(\mathrm{p}<0.001)$. Grade $3 \geq$ positivity in salivary gland biopsy was found as $71.2 \%$ in primary Sjögren's syndrome group and $27.2 \%$ in the undifferentiated group $(\mathrm{p}<0.001)$.

Conclusions: More dense lymphocyte infiltration was detected in primary Sjögren's syndrome in salivary gland biopsies. As $27.2 \%$ of undifferentiated group patients had positive salivary gland biopsies, they might need follow-ups in terms of having increased risk of developing primary Sjögren's syndrome.

Keywords: Keratoconjuctivitis sicca, Schirmer's test, Sjögren's syndrome
\end{abstract}

\section{INTRODUCTION}

Sjögren's syndrome (SS) is a chronic autoimmune disease of unknown cause, mainly involving the salivary gland and lacrimal gland. Primary Sjögren's syndrome (PSS) is one of the three most common autoimmune disorders in the population, having an average incidence of $0.5-1.0 \%$. The disorder may be confined only to the salivary and lacrimal glands, or in one-third of the cases, it may be associated with various organ/system involvements such as musculoskeletal, gastrointestinal, nervous systems and skin, kidneys, lungs, and lymph nodes. Symptoms such as fatigue and arthralgia, which severely affect patients' quality of life, are frequently identified beside the objective signs. SS can occur alone or with rheumatological diseases such as rheumatoid arthritis (RA) and systemic lupus erythematosus
(SLE). Such a situation is named secondary Sjögren's syndrome (SSS) $[1,2]$.

Keratoconjunctivitis sicca (dry eye) is a disease in which eye complaints are observed with an increased frequency with advanced age. In this case, biochemical and serologic markers are negative. Even though common in the population, it is, unfortunately, a neglected disease group because patients do not perceive its symptoms adequately and physicians do not recognize its systemic findings well [3].

The presence of systemic involvement and some other serological and biochemical markers are important indicators for the disease progression. For this reason, patients' clinical and

How to cite this article: Taskiran I, Kalyoncu U, Kiraz S. Clinical and biochemical characteristics of Sjögren's syndrome and its differences from keratoconjunctivitis sicca. Marmara Med J 2021; 34(2):208-214. doi: 10.5472/marumj.944279 
biochemical characteristics at first admission have some clues about how the disease will progress in the early period.

Our study aimed to determine the demographic, clinical, and laboratory features of patients followed up for SS. Its other aim was to determine the SS group's differences from the keratoconjunctivitis sicca and undifferentiated groups.

\section{PATIENTS and METHODS}

The study's patient group comprised the patients followed up with the diagnoses of Sjögren's syndrome (primary and secondary) and keratoconjunctivitis sicca (dry eye) in the Rheumatology Department of a university hospital between August 2009 and January 2010. In this context, the newly diagnosed patients and the previously diagnosed patients who presented for a control examination were included in the study. Patients who did not consent and those who did not accept to undergo salivary gland biopsy were excluded from the study. The patient age and gender, ages at diagnosis and the onset of symptoms, initial symptoms, smoking status, physical examination findings such as blood pressure, articular features (arthritis/arthralgia), Raynaud phenomenon, lymphadenopathy, and laboratory parameters such as complete blood count, liver and renal function tests, erythrocyte sedimentation rate (ESR), C-reactive protein (CRP), rheumatoid factor (RF), complement 3-4 (C3-C4), urinalysis, thyroid tests - thyroid autoantibodies, antinuclear antibody (ANA), anti-Ro, anti-La, anti double stranded DNA (anti-ds DNA), lupus anticoagulant, antiphospholipid and anticardiolipin antibodies, hepatitis markers, and the gammaglobulin ratio in serum protein electrophoresis were recorded. The evaluation of salivary gland biopsy was performed according to the ChisolmMason criteria [4]. Biopsy results revealing Grade 3 and over were considered as diagnostic for SS. (Lymphocytes per $4 \mathrm{~mm} 2$ / salivary tissue. Grade 0: absent, grade 1: slight infiltration, grade 2: moderate infiltration or less than one focus, grade 3 : one focus, grade 4: more than one focus. Focus: aggregate of $\geq$ 50 lymphocytes and histiocytes). When present, the patients' parotid scintigraphy results were recorded.

The patients were queried thoroughly regarding dry mouth and eye, the primary symptoms of SS. Dry mouth and dry eye were assessed as present/absent. The symptoms were classified as mild, moderate, severe, and highly severe in patients with dry mouth/eye. While classifying the symptoms of dry mouth and eyes of the patients, the patients were asked and scored from 1 to 10 according to the severity of the complaints. (1-4 points: mild, 5-6 points: moderate, 7-8 points: severe, 9-10 points: highly severe). The dry mouth/eye symptoms were recorded as between 0-100 mm using the Visual Analog Scale (VAS), and the symptom durations were also recorded. Fatigue, a significant SS symptom, was recorded as present/absent, like dry mouth/eye. Then, VAS was used to record its severity between 0-100 mm.

Schirmer's test was performed at the Outpatient Ophthalmology Clinic, and test results under $5 \mathrm{~mm}$ were considered positive [5].

The imaging methods (computed tomography, magnetic resonance imaging) guided the investigations, especially regarding the lung and the central nervous system involvements and serologic markers for hepatic involvement.

The diagnosis of vasculitis was verified by discovering palpable purpura on physical examination and identifying leukocytoclastic vasculitis in skin biopsies.

The patients' medications, such as hydroxychloroquine, corticosteroids, methotrexate, sulphasalazine, and acetylsalicylic acid, and their dosages were recorded.

The accompanying rheumatologic diseases, such as RA, SLE, and scleroderma, were recorded. Besides, other disorders that might have been associated, such as autoimmune thyroiditis, autoimmune hepatitis, and primary biliary cirrhosis, were recorded.

In this study, patients were divided into 4 different groups. The patients with four of the SS criteria were defined as 'primary' SS, whereas, those with positive for three criteria or less as 'undifferentiated' group. Patients who fulfilled the diagnostic criteria for SS and had additional rheumatologic disease were defined as SSS. Only patients with dry eye complaints and negative biochemical/serological markers were evaluated as keratoconjunctivitis sicca (dry eye).

The diagnosis of SS was made according to the criteria defined in 2002 [6].

This study was approved by the Ethics Committee of a university hospital where the study was conducted (approval number: HEC 09/91-227).

\section{Statistical Analysis}

Statistical analysis was performed by the "Statistical Package for Social Sciences (SPSS) v. 15.0" software. Data were presented as mean and standard deviation. The Student's t-test was used for continuous variables and the Chi-square test for categorical variables. A "p" value under 0.05 was considered statistically significant.

\section{RESULTS}

This study evaluated a total of 183 patients. Eighty-seven patients fulfilled the criteria for PSS diagnosis, whereas 25 patients met the criteria for diagnosing SSS. When patients with SSS were analyzed regarding rheumatologic disorders, it was found that 11 patients (44\%) had RA, 13 (52\%) SLE, and one (4\%) patient had spondyloarthropathy. In 27 patients, only dry eye findings were present. In 44 patients, the diagnostic criteria of SS were not fulfilled; however, they were defined as the "undifferentiated group" because of their symptom/antibody positivity or a positive result in salivary gland biopsy.

All patients' demographic data and clinical features/organ involvement were presented in Table I. In all four groups, more than $85 \%$ of the patients were females. In all groups, the mean patient age was within the $4^{\text {th }}$ decade. No significant differences were present among the four groups regarding the present age and the age at the onset of symptoms ( $p>0.05)$. 
Table I: Demographic and clinical characteristics of the patients in the study

\begin{tabular}{|c|c|c|c|c|}
\hline & $\begin{array}{c}\text { Primary SS } \\
(\mathrm{n}=\mathbf{8 7})\end{array}$ & $\begin{array}{c}\text { SSS } \\
(\mathrm{n}=25)\end{array}$ & $\begin{array}{l}\text { Dry eye } \\
(n=27)\end{array}$ & Undifferentiated $(\mathrm{n}=44)$ \\
\hline Age (years $\pm S D)$ & $48 \pm 13$ & $49 \pm 14$ & $45 \pm 12$ & $47 \pm 11$ \\
\hline \multicolumn{5}{|l|}{ Gender n(\%) } \\
\hline Female & $83(95)$ & $22(88)$ & $23(85)$ & $40(91)$ \\
\hline Male & $4(5)$ & $3(12)$ & $4(15)$ & $4(9)$ \\
\hline Disease duration (years) & $1(0-20)^{2}$ & $3(0-26)^{2}$ & $1(0-10)^{2}$ & $2(0-15)^{2}$ \\
\hline \multicolumn{5}{|l|}{$\begin{array}{l}\text { Severity of dry mouth } \\
\mathrm{n}(\%)\end{array}$} \\
\hline Absent & $3(3.4)$ & $1(4)$ & $14(51.8)$ & $19(43.2)$ \\
\hline Mild & $17(19.5)$ & $4(16)$ & $4(14.8)$ & $7(15.9)$ \\
\hline Moderate & $28(32.2)$ & $11(44)$ & $7(26.0)$ & $10(22.7)$ \\
\hline Severe & $37(42.5)$ & $7(28)$ & $2(7.4)$ & $7(15.9)$ \\
\hline Highly severe & $2(2.3)$ & $2(8)$ & $0(0)$ & $1(2.3)$ \\
\hline Mouth VAS mm & $53 \pm 22^{1}$ & $54 \pm 21^{1}$ & $0(0-80)^{2}$ & $25(0-90)^{2}$ \\
\hline \multicolumn{5}{|l|}{ Severity of dry eye } \\
\hline \multicolumn{5}{|l|}{$\mathrm{n}(\%)$} \\
\hline Absent & $1(1.1)$ & $3(12)$ & $1(3.7)$ & $17(38.6)$ \\
\hline Mild & $19(21.8)$ & $3(12)$ & $1(3.7)$ & $6(13.6)$ \\
\hline Moderate & $30(34.5)$ & $4(16)$ & $4(14.8)$ & $15(34.1)$ \\
\hline Severe & $34(39.1)$ & $13(52)$ & $16(59.3)$ & $6(13.6)$ \\
\hline Highly severe & $3(3.4)$ & $2(8)$ & $5(18.5)$ & $0(0)$ \\
\hline Eye VAS mm & $53 \pm 21^{1}$ & $49 \pm 30^{1}$ & $67 \pm 18^{1}$ & $28 \pm 27^{1}$ \\
\hline Schirmer's test mm & $4(1-35)^{2}$ & $5(1-35)^{2}$ & $3.2 \pm 1.9^{1}$ & $13.4 \pm 9.1^{1}$ \\
\hline Fatigue $\mathrm{n}(\%)$ & $70(80.5)$ & $22(88)$ & $19(70.4)$ & $34(77.3)$ \\
\hline Fatigue VAS mm & $50 \pm 27^{1}$ & $53 \pm 22^{1}$ & $42 \pm 30^{1}$ & $45 \pm 28^{1}$ \\
\hline Arthralgia n (\%) & $63(74.1)$ & $24(96.0)$ & $18(66.7)$ & $32(72.7)$ \\
\hline Arthritis n (\%) & $11(12.9)$ & $17(68.0)$ & $2(7.4)$ & $5(11.4)$ \\
\hline Raynaud phenomenon n (\%) & $12(14.1)$ & $3(12.0)$ & $0(0)$ & $6(13.6)$ \\
\hline Vasculitis n (\%) & $1(1.2)$ & $2(8)$ & $0(0)$ & $2(4.5)$ \\
\hline Neurologic involvement n (\%) & $2(2.4)$ & $2(8.0)$ & $2(7.4)$ & $3(6.8)$ \\
\hline Transverse myelitis & 1 & 0 & 0 & 0 \\
\hline Optic neuritis + T. Myelitis & 1 & 0 & 0 & 0 \\
\hline Optic atrophy & 0 & 1 & 0 & 0 \\
\hline Peripheral neuropathy & 0 & 0 & 0 & 1 \\
\hline Myasthenia Gravis & 0 & 1 & 0 & 0 \\
\hline Demyelinating Disease & 0 & 0 & 2 & 0 \\
\hline Ischemic Changes & 0 & 0 & 0 & 2 \\
\hline Lung Involvement n (\%) & $6(7.2)$ & $3(12)$ & $0(0)$ & $0(0)$ \\
\hline Interstitial & 1 & 0 & 0 & 0 \\
\hline Ground glass & 2 & 2 & 0 & 0 \\
\hline Pulmonary nodule & 2 & 0 & 0 & 0 \\
\hline Honeycomb + ground glass & 1 & 0 & 0 & 0 \\
\hline $\begin{array}{l}\text { Ground glass + pulmonary } \\
\text { nodule }\end{array}$ & 0 & 1 & 0 & 0 \\
\hline Liver n (\%) & $4(4.6)$ & $0(0)$ & $0(0)$ & $1(2.3)$ \\
\hline Autoimmune hepatitis & 3 & 0 & 0 & 1 \\
\hline PBS & 1 & 0 & 0 & 0 \\
\hline Autoimmune thyroiditis n (\%) & $10(11.4)$ & $3(12)$ & $3(11)$ & $3(6.8)$ \\
\hline
\end{tabular}

Data were presented as mean \pm SD or median (min-max).

The average value was shown in the table as ${ }^{1}$ and the median value as ${ }^{2}$.

PBS: Primary biliary cirrhosis 
In PSS patients with severe and highly severe mouth dryness, biopsy results revealing Grades 3 and 4 were more common in the minor salivary gland biopsies $(\mathrm{p}=0.003)$ (Table II).

Table II. The grades of minor salivary gland biopsy according to the severity of dry mouth in PSS patients

\begin{tabular}{lcc} 
& $\begin{array}{c}\geq \text { Severe dry mouth } \\
(\mathrm{n}=39)\end{array}$ & $\leq$ Moderate dry mouth $(\mathrm{n}=48)$ \\
\hline Grade $0 \mathrm{n}(\%)$ & $4(10.2)$ & $3(6.2)$ \\
Grade $1 \mathrm{n}(\%)$ & $2(5.1)$ & $8(16.7)$ \\
Grade 2 $\mathrm{n}(\%)$ & $1(2.6)$ & $7(14.6)$ \\
Grade 3n $(\%)$ & $17(43.5)$ & $26(54.2)$ \\
Grade 4n $(\%)$ & $15(38.6)$ & $4(8.3)$ \\
\hline
\end{tabular}

Positive results in salivary gland biopsies were less frequent in patients with severe and highly severe dry eyes (56.8\%) when compared to those with mild and moderate dry eyes $(82.0 \%)$ $(\mathrm{p}=0.01)$ (Table III).

Table III. The grades of minor salivary gland biopsy according to the severity of dry eye in PSS patients

\begin{tabular}{|ccc|}
\hline & $\geq$ Severe dry eye $(\mathrm{n}=37)$ & $\leq$ Moderate dry eye $(\mathrm{n}=50)$ \\
\hline Grade $0 \mathrm{n}(\%)$ & $3(8.1)$ & $4(8.0)$ \\
Grade $1 \mathrm{n}(\%)$ & $7(18.9)$ & $3(6.0)$ \\
\hline Grade 2 $\mathrm{n}(\%)$ & $6(16.2)$ & $2(4.0)$ \\
Grade 3 $\mathrm{n}(\%)$ & $11(29.8)$ & $32(64.0)$ \\
Grade $4 \mathrm{n}(\%)$ & $10(27.0)$ & $9(18.0)$ \\
\hline
\end{tabular}

The results of laboratory parameters were presented in Table IV. There was a history of cardiac pacemaker implantation in two patients with PSS because of a heart block due to anti-Ro antibodies. A similar situation was determined in a patient with the undifferentiated disease.

Non-Hodgkin lymphoma (NHL) was diagnosed in two patients with PSS. The pathological typing was large cell B-cell lymphoma in both patients, and one of them was CD20(+). Both patients had pathological-sized lymphadenopathies. One of the patients was diagnosed with leukocytoclastic vasculitis accompanying SS. NHL developed ten years after the patient had been diagnosed with SS. The other patient was also diagnosed with NHL accompanying SS. This patient had leukopenia and hepatosplenomegaly at admission.

In $70(80.4 \%)$ of PSS patients, $21(84 \%)$ of SSS patients, and $25(56.8 \%)$ of patients with the undifferentiated disease, the salivary gland biopsy revealed the severity as Grade 2 and above. No patient had a salivary gland biopsy result of Grade 2 and above in the dry eye group (Table V). A Grade 3 and over salivary gland biopsy result was present in 62 of 87 PSS patients. Severe and highly severe dry mouth was present in 32 (51.6\%) of these 62 patients, whereas in $7(28.0 \%)$ of 25 patients with a grade between 0 and $2(\mathrm{p}=0.01)$ (Table II).
When the PSS patients were compared to the patients with a dry eye only, significant differences were determined regarding clinical findings (mouth-eye dryness severity and VAS scores, Raynaud phenomenon), laboratory parameters (ESR, globulin, RF, ANA, anti-Ro, anti-La), and minor salivary gland biopsy results. While the severity of mouth dryness and laboratory investigations indicated PSS, the severity of eye dryness and Schirmer's test's frequency and severity were more significantly determined in the group with a dry eye only (Table VI).

Table IV. Laboratory results of the patients

\begin{tabular}{|c|c|c|c|c|}
\hline & $\begin{array}{c}\text { Primary } \\
\text { SS } \\
(n=87)\end{array}$ & $\begin{array}{c}\text { SSS } \\
(\mathrm{n}=25)\end{array}$ & $\begin{array}{l}\text { Dry eye } \\
(\mathrm{n}=27)\end{array}$ & $\begin{array}{l}\text { Undifferentiated } \\
\qquad(\mathrm{n}=44)\end{array}$ \\
\hline \multicolumn{5}{|l|}{ ANA n (\%) } \\
\hline Absent & $8(9.2)$ & $3(12.0)$ & $21(77.8)$ & $7(15.9)$ \\
\hline $1 / 80$ & $16(18.4)$ & $4(16.0)$ & $4(14.8)$ & $5(11.4)$ \\
\hline $1 / 160$ & $42(48.3)$ & $8(32.0)$ & $2(7.4)$ & $16(36.4)$ \\
\hline $1 / 320$ & $19(21.8)$ & $7(28.0)$ & $0(0)$ & $14(31.8)$ \\
\hline $1 / 640$ & $2(2.3)$ & $3(12.0)$ & $0(0)$ & $2(4.5)$ \\
\hline RF n (\%) & $43(54.0)^{3}$ & $14(56.0)$ & $6(22.3)$ & $14(31.8)$ \\
\hline Anti-Ro n (\%) & $55(62.5)$ & $15(60.0)$ & $0(0)$ & 27 (63.6) \\
\hline Anti-La n (\%) & $30(34.1)$ & $8(32.0)$ & $0(0)$ & $14(31.8)$ \\
\hline Anti-TPO n (\%) & $8(9.1)$ & $2(8.0)$ & $3(11)$ & $2(4.5)$ \\
\hline $\begin{array}{l}\text { Anti-thyroglobulin } \\
\mathrm{n}(\%)\end{array}$ & $9(10.2)$ & $1(4.0)$ & $3(11)$ & $2(4.5)$ \\
\hline Hemoglobin gr/dl & $12.8 \pm 1.4^{1}$ & $12.8 \pm 1.6^{1}$ & $13.7 \pm 1.6^{1}$ & $13.1 \pm 1.4^{1}$ \\
\hline $\begin{array}{l}\text { Leukocyte } \mathrm{mm}^{3} \\
\mathrm{x} 10^{3}\end{array}$ & $6.2 \pm 2.4^{1}$ & $6.5 \pm 2.1^{1}$ & $7.6 \pm 2.3^{1}$ & $6.1 \pm 2.6^{1}$ \\
\hline $\begin{array}{l}\text { Thrombocyte } \mathrm{mm}^{3} \\
\mathrm{x} 10^{3}\end{array}$ & $268 \pm 73^{1}$ & $269 \pm 98^{1}$ & $234 \pm 40^{1}$ & $258 \pm 61.7^{1}$ \\
\hline ESR mm/hour & $27 \pm 21^{1}$ & $42 \pm 30^{1}$ & $10 \pm 7$ & $25 \pm 20$ \\
\hline CRP mg/dl & $\begin{array}{c}0.3 \\
(0.1-21)^{2}\end{array}$ & $\begin{array}{c}0.8 \\
(0.1-9,2)^{2}\end{array}$ & $\begin{array}{c}0.30 \\
(0.1-1.6)^{2}\end{array}$ & $\begin{array}{c}0.27 \\
(0.1-11)^{2}\end{array}$ \\
\hline $\begin{array}{l}\text { Hyperglobulinemia } \\
\mathrm{n}(\%)\end{array}$ & $10(12.6)$ & $2(8)$ & $0(0)$ & $2(4.6)$ \\
\hline
\end{tabular}

Data were presented as mean $\pm S D$ or median (min-max).

The average value was shown in the table as ${ }^{1}$ and the median value as ${ }^{2}$.

${ }^{3} R F$ was evaluated with 80 patients. ANA: antinuclear antibody, RF: rheumatoid factor, Anti-TPO: anti thyroid peroxidase antibody, ESR: erythrocyte sedimentation rate, $C R P$ : $C$ reactive protein

Contrary to the dry eye patients, fewer differences were determined between the PSS and undifferentiated groups. When the PSS patients were compared to the patients with the undifferentiated disease, while significant differences were determined regarding clinical findings (mouth-eye dryness severity and VAS scores) and minor salivary gland biopsy results, the laboratory tests of the two groups revealed similar results (Table VII). 
The patients' treatment characteristics were presented in Table VIII.

Table V. Salivary gland biopsy results of the patients

\begin{tabular}{lcccc} 
& $\begin{array}{c}\text { Primary SS } \\
\mathbf{n}(\%)\end{array}$ & $\begin{array}{c}\text { SSS } \\
\mathbf{n}(\%)\end{array}$ & $\begin{array}{c}\text { Dry eye } \\
\mathbf{n}(\%)\end{array}$ & $\begin{array}{c}\text { Undifferentiated } \\
\mathbf{n}(\%)\end{array}$ \\
\hline Grade 0 & $7(8.1)$ & $2(8)$ & $19(70.4)$ & $10(22.7)$ \\
Grade 1 & $10(11.5)$ & $2(8)$ & $8(29.6)$ & $9(20.5)$ \\
Grade 2 & $8(9.2)$ & $5(20)$ & $0(0)$ & $13(29.5)$ \\
Grade 3 & $43(49.4)$ & $10(40)$ & $0(0)$ & $10(22.7)$ \\
Grade 4 & $19(21.8)$ & $6(24)$ & $0(0)$ & $2(4.5)$ \\
\hline
\end{tabular}

Table VI. The differences between primary SS and dry eye

\begin{tabular}{lccc}
\hline & Primary SS & Dry Eye & p \\
$\begin{array}{l}\text { Severe/highly severe dry mouth } \\
\mathrm{n}(\%)\end{array}$ & $39(45)$ & $2(7)$ & 0.001 \\
\hline Dry mouth VAS (mm) mean \pm SD & $53 \pm 22$ & $21 \pm 25$ & $<0.001$ \\
\hline Schirmer (mm) mean \pm SD & $8.7 \pm 8.6$ & $3.3 \pm 1.9$ & 0.002 \\
\hline Schirmer positivity n (\%) & $61(70)$ & $27(100)$ & 0.002 \\
\hline Dry eye VAS (mm) mean \pm SD & $53 \pm 21$ & $67 \pm 17$ & 0.001 \\
ESR (mm/hour) mean \pm SD & $27 \pm 21$ & $10 \pm 7$ & $<0.001$ \\
\hline Globulin (units) mean \pm SD & $3.69 \pm 0.63$ & $2.94 \pm 0.35$ & $<0.001$ \\
Severe/highly severe dry eye n (\%) & $37(43)$ & $21(78)$ & $<0.001$ \\
\hline RF > 40 IU n (\%) & $43(54)$ & $3(11)$ & $<0.001$ \\
\hline $\begin{array}{l}\text { ANA } \geq 1 / 160 \text { titration positivity } \\
\text { n (\%) }\end{array}$ & $63(72)$ & $2(7)$ & $<0.001$ \\
Anti-Ro n (\%) & $55(62)$ & $0(0)$ & $<0.001$ \\
Anti-La n (\%) & $30(34)$ & $0(0)$ & $<0.001$ \\
\hline $\begin{array}{l}\text { Raynaud Phenomenon n (\%) } \\
\text { Salivary gland biopsy } \geq \text { grade 3 }\end{array}$ & $12(14)$ & $0(0)$ & 0.028 \\
n (\%) & $62(71)$ & $0(0)$ & $<0.001$ \\
\hline
\end{tabular}

VAS: visual analog scale, ESR: erythrocyte sedimentation rate, RF: rheumatoid factor, ANA: antinuclear antibody

Table VII. The differences between primary SS and undifferentiated groups

\begin{tabular}{lccc} 
& $\begin{array}{c}\text { Primary } \\
\text { SS } \\
(\mathbf{n}=\mathbf{8 7})\end{array}$ & $\begin{array}{c}\text { Undifferentiated } \\
(\mathbf{n}=\mathbf{4 4})\end{array}$ & $\mathbf{p}$ \\
\hline $\begin{array}{l}\text { Severe/highly severe dry mouth } \\
\mathrm{n}(\%)\end{array}$ & $39(45)$ & $8(18)$ & 0.003 \\
$\begin{array}{l}\text { Dry mouth VAS (mm) } \\
\text { Schirmer positivity n (\%) }\end{array}$ & $53 \pm 21$ & $28 \pm 29$ & $<0.001$ \\
$\begin{array}{l}\text { Dry eye VAS (mm) } \\
\text { Severe/highly severe dry eye }\end{array}$ & $37(40)$ & $11(25)$ & $<0.001$ \\
$\begin{array}{l}\text { n (\%) } \\
\text { Salivary gland biopsy }(+) \mathrm{n}(\%)\end{array}$ & $62(71)$ & $6(14)$ & 0.001 \\
\hline
\end{tabular}

Table VIII. Treatment features of all patients included in the study

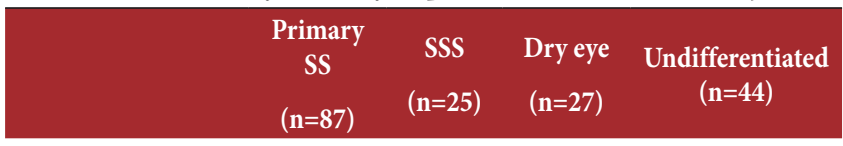

Hydroxychloroquine

$\mathrm{n}(\%)$

\begin{tabular}{lcccc}
$200 \mathrm{mg}$ & $27(30.7)$ & $6(24.0)$ & $0(0)$ & $9(20.5)$ \\
$400 \mathrm{mg}$ & $54(61.4)$ & $18(72.0)$ & $0(0)$ & $32(72.7)$ \\
Corticosteroid mg & 5.5 & 6.75 & $0(0)$ & 3.25 \\
& $(2-1000)^{1}$ & $(1-40)^{1}$ & & $(2-1000)^{1}$ \\
Methotrexate n (\%) & & & & \\
$7,5 \mathrm{mg} /$ week & $0(0)$ & $2(8)$ & $0(0)$ & $0(0)$ \\
$10 \mathrm{mg} /$ week & $3(3.4)$ & $2(8)$ & $0(0)$ & $2(4.5)$ \\
$15 \mathrm{mg} /$ week & $0(0)$ & $5(20)$ & $0(0)$ & $1(2.3)$ \\
Azathioprine n (\%) & & & & \\
$50 \mathrm{mg}$ & $2(2.3)$ & $2(8)$ & $0(0)$ & $1(2.3)$ \\
$100 \mathrm{mg}$ & $13(14.8)$ & $5(20)$ & $0(0)$ & $4(9.1)$ \\
$150 \mathrm{mg}$ & $2(2.3)$ & $0(0)$ & $0(0)$ & $0(0)$ \\
Sulfasalazine n (\%) & $0(0)$ & $9(36)$ & $0(0)$ & $2(4.5)$ \\
Cyclosporin n (\%) & $1(1.1)$ & $0(0)$ & $0(0)$ & $1(2.2)$ \\
\hline
\end{tabular}

\section{DISCUSSION}

In our study, a total of 183 patients were evaluated, involving 87 PSS, 25 SSS, 27 keratoconjunctivitis sicca patients, and 44 patients with the undifferentiated disease. No significant differences were present regarding age and gender among these groups. The onset of the disease was in the $4^{\text {th }}$ decade in all groups.

Evaluating the dry mouth and dry eye using a Likert scale is not a frequently performed practice in SS. These symptoms have generally been defined as present or absent. In fact, dry mouth and dry eye symptoms were defined as present/absent during diagnostic criteria constitution. However, they were classified as absent, mild, moderate, severe, and highly severe in our study. We think that such a classification reflects the patient symptomatology better. Actually, the salivary gland biopsies of the patients with severe and highly severe dry mouth symptoms revealed positive results more commonly.

In daily practice, the number of patients with dry mouth and/or eye, a positive/negative result in Schirmer's test, and a positive result for any antibody is not small. It is not uncommon to identify lymphocytic infiltration of Grade 2 and over in biopsies of the minor salivary glands in these patients (undifferentiated group). In this patient group, $29.5 \%$ of patients had Grade 2 lymphocytic infiltration, whereas, in $27.2 \%$ of patients, it was Grade 3 and over. For this reason, even though these patients could not fulfill the diagnostic criteria of PSS, they had many subjective and objective findings. We defined this group as the group "not entirely differentiated to PSS." The undifferentiated group was similar to the PSS group regarding age and gender. 
While there was no dry mouth symptom in more than $40 \%$ of this group, severe and highly severe dry mouth was present in only $18 \%$ of the group. Like the dry mouth symptom, dry eye was not identified in $38 \%$ of the patients, and only $14 \%$ had highly severe dry eye symptoms. In this group, $77 \%$ of the patients had fatigue as one of the primary symptoms. There were no differences between the PSS and undifferentiated groups regarding arthralgia, Raynaud phenomenon, arthritis, neurological involvement, autoimmune thyroiditis, vasculitis, and autoimmune hepatic involvement. Fatigue is one of the major complaints in rheumatological diseases. A study investigating the quality of life in PSS determined that patients had encountered a 5-65\% reduction in quality of life [7-8]. We used a $100 \mathrm{~mm}$ VAS, commonly used in the literature, as a practical and straightforward measurement method. Even though it might reveal false-positive and false-negative results according to patients' socioeconomic levels, it is a test commonly used today. In our study, $80 \%$ of PSS patients had fatigue, and this ratio was a little higher than those in the literature.

Auto-antibodies are among the most significant objective criteria in diagnosing SS. Various studies have reported auto-antibody positivities in PSS as follows: RF: 6-60\%, ANA: around 90\%, anti-Ro: 23-60\%, anti-La: 5-50\% [9]. In our study, ANA $\geq 1 / 160$ titration was present in $72.4 \%$ of PSS patients, whereas $18.4 \%$ of the patients had an ANA positivity at the titration of $1 / 80$. RF was present in 54\%, anti-Ro in 62\%, and anti-La antibody in $34 \%$ of the patients. These results were consistent with those in the literature. The autoantibodies were absent in the dry eye patients. Intriguingly, there was no difference between the PSS and undifferentiated groups regarding autoantibody commonness. The high auto-antibody positivity in the undifferentiated group might have been evidence of the close relationship of this group with the PSS group.

PSS is 10 -fold more common in patients with autoimmune thyroiditis. Similarly, autoimmune thyroiditis is 9-fold more frequently met in PSS patients. In a study conducted in 1998 by Gaches et al. on 218 patients with autoimmune thyroiditis [10], the most common autoimmune disorders were reported as SLE and SS. In another recent study, the close coexistence of the two conditions was reported [11]. In our patients, autoimmune thyroid disease was determined with a frequency of $11.4 \%$ in PSS. However, autoimmune thyroid disease had a high ratio in the SSS, dry eye, and undifferentiated groups. In our SSS patients, such a high ratio is not surprising because RA and SLE disorders have been well-known to lead to autoimmune thyroiditis.

On the other hand, the rate of $6.8 \%$ for autoimmune thyroiditis in the undifferentiated group might support the fact that the undifferentiated group was a sub-group of PSS. In our study, 3 of 27 dry eye patients had autoimmune thyroiditis. The relationship between autoimmune thyroid diseases and dry eye is a subject that necessitates further research.

Raynaud's phenomenon is a vascular disorder characterized by episodes of reversible digital ischemia in response to cold or stress. Raynaud's phenomenon is generally classified as primary (in the absence of any associated process) or secondary if an associated disease (most frequently an autoimmune disease) or an environmental condition is present. Raynaud phenomenon has been reported with a frequency between $13-38 \%$ in previous studies [12]. In our study, the incidence of the Raynaud's phenomenon was $14.1 \%$ in PSS patients, similar to those in the literature.

In our study, in two (2.3\%) PSS patients and in one patient in the undifferentiated group, congenital heart block due to blocking antibodies (anti-Ro/anti-La) was determined, and this result was similar to the literature [13-14].

Various studies have reported that the lymphoma risk was increased in PSS patients $[15,16]$. In our study, two $(2.3 \%)$ PSS patients had lymphoma. One patient had persistent hepatosplenomegaly leukopenia and lymphadenopathy, whereas another patient had cutaneous vasculitis and lymphadenopathy in our study.

The dry eye symptom is mostly at the forefront among the causes for admission of PSS patients to Rheumatology Clinics. There is a significant patient population with only dry eyes followed up at ophthalmology clinics. The differential diagnosis of PSS with dry eye is crucial. In our study, the clinical and laboratory results suggesting PSS were determined as the severity of mouth dryness, Raynaud phenomenon, high ESR, elevated globulin level, and positivity of RF, ANA, anti-Ro, and anti-La antibodies. The frequency of positive results in Schirmer's test and more severe eye dryness were determined as the characteristics of the dry eye group. It is not always possible to differentiate the two conditions clinically and by laboratory methods. In such conditions, salivary gland biopsy is a beneficial method. No biopsy result of Grade 2 and over was encountered in patients with dry eyes.

In this study, the most common treatment that the PSS patients received was hydroxychloroquine. Besides this treatment, low-dose glucocorticoids were administered. Azathioprine, cyclophosphamide, and methotrexate were used in the patients with extraglandular involvement. The patients in the PSS and undifferentiated groups received similar treatments. Because our study was not designed as a follow-up study, we could not further interpret the treatment's efficacy.

The evaluation of symptoms, Schirmer's tests, auto-antibody investigations, and salivary gland biopsies were performed in all patients. On the other hand, the parotid scintigraphy was not evaluated in every patient, which was our study's most significant limitation. The patients positive for four of the SS criteria were defined as "definite" SS, whereas those positive for three criteria or less as "undifferentiated" group. One of these six criteria was identifying the parotid gland involvement by an objective test. The most commonly used method for this purpose is parotid gland scintigraphy in daily practice. However, we performed the scintigraphic investigation in only $8.7 \%$ of our patients. The cause of such a low rate for scintigraphic evaluation was that the salivary gland biopsy was an easily applicable investigation that could be performed in outpatient clinics. 


\section{Conclusion}

The severity of mouth and eye dryness was more significant, and more dense lymphocytic infiltration was identified in biopsies in PSS patients than patients in the undifferentiated group. Severe ocular symptoms were present in dry eye patients when compared to PSS patients. Because the salivary gland biopsy revealed positive results in $27.2 \%$ of patients in the undifferentiated group, follow-up of these patients would be required regarding PSS development.

\section{Compliance with Ethical Standards}

Ethics approval: This study was approved by the Ethics Committee of a university hospital where the study was conducted (approval number: HEC 09/91-227).

Funding: The authors have no relevant financial information to disclose.

Conflict of Interest: The authors declare no conflict of interest. Authors' Contributions: Literature search: IT, Study design: IT and UK, Legislative applications: IT, Data collection: IT, Supervision and quality control: SK and UK, Statistical advice: UK, Statistical data analysis: UK, Data interpretation: IT, UK and SK, Drafting the manuscript: IT. All authors read and approved the final version of the article.

\section{REFERENCES}

[1] Jonsson R, Bowman SJ, Gordon TP. Sjögren's syndrome. In: Koopman WJ, ed. Arthritis and Allied Conditions. Philadelphia: Lippincott Williams and Wilkins, 2005: 1681705.

[2] Delaleu N, Jonsson R, Koller MM. Sjogren's syndrome. Eur J Oral Sci 2005;113:101-13.doi:10.1111/j.16000722.2004.00183.x

[3] Strickland RW, Tesar JT, Berne BH, Hobbs BR, Lewis DM, Welton RC. The frequency of sicca syndrome in an elderly female population. J Rheumatol 1987;14:766-71

[4] Chisholm DM, Mason DK. Labial salivary gland biopsy in Sjögren's disease. J Clin Pathol 1968;21:656-60.

[5] Stevens S. Schirmer's test. Community Eye Health 2011;24:45.

[6] Vitali C, Bombardieri S, Jonsson R, Moutsopoulos HM, Alexander EL, Carsons SE, et al. Classification criteria for Sjogren's syndrome: a revised version of the European criteria proposed by the American-European Consensus Group. Ann Rheum Dis 2002;61:554-8.doi:10.1136/ard.61.6.554
[7] Strombeck B, Ekdahl C, Manthorpe R, Wikstrom I, Jacobsson L. Health-related quality of life in primary Sjogren's syndrome, rheumatoid arthritis and fibromyalgia compared to normal population data using SF-36. Scand J Rheumatol 2000;29:20-8. doi:10.1080/030.097.400750001761

[8] Carsons SE, Vivino FB, Parke A, et al. Treatment guidelines for rheumatologic manifestations of Sjögren's syndrome: Use of biologic agents, management of fatigue, and inflammatory musculoskeletal pain. Arthritis Care Res 2017;69:517-27. doi. org/10.1002/acr.22968

[9] Diaz-Lopez C, Geli C, Corominas H, Malat N, Diaz-Torner C, Llobet JM, et al. Are there clinical or serological differences between male and female patients with primary Sjogren's syndrome? J Rheumatol 2004;31:1352-5

[10] Gaches F, Delaire L, Nadalon S, Loustaud-Ratti V, Vidal E. [Frequency of autoimmune diseases in 218 patients with autoimmune thyroid pathologies]. Rev Med Interne 1998;19:173-9.doi:10.1016/s0248-8663(97)80716-3

[11] Anaya JM, Jimenez PR, Rodriguez Y, et al. Sjögren's syndrome and autoimmune thyroid disease: Two sides of the same coin. Clin Rev Allergy Immunol 2019;56:362-74. doi: 10.1007/ s12016.018.8709-9.

[12] Garcia-Carrasco M, Siso A, Ramos-Casals M, Rosas J, de la Red G, Gil V, et al. Raynaud's phenomenon in primary Sjogren's syndrome. Prevalence and clinical characteristics in a series of 320 patients. J Rheumatol 2002;29:726-30

[13] Schmidt KG, Ulmer HE, Silverman NH, Kleinman CS, Copel JA. Perinatal outcome of fetal complete atrioventricular block: a multicenter experience. J Am Coll Cardiol 1991;17:1360-6. doi:10.1016/s0735-1097(10)80148-2

[14] Gupta S, Gupta N. Sjögren syndrome and pregnancy: A litearature review. Perm J 2017;21:16-047. doi: 10.7812/ TPP/16-047.

[15] Zhang W, Feng S, Yan S, Zhao Y, Li M, Sun J, et al. Incidence of malignancy in primary Sjogren's syndrome in a Chinese cohort. Rheumatology (Oxford) 2010;49:571-7.doi:10.1093/ rheumatology/kep404

[16] Fragkioudaki S, Mavragani CP, Moutsopoulos HM. Predicting the risk for lymphoma development in Sjogren syndrome: An easy tool for clinical use. Medicine (Baltimore) 2016;95:e3766. doi:10.1097/MD.000.000.0000003766 